\title{
SDO Onboard Ephemeris Generation
}

\author{
Kevin E. Berry ${ }^{1}$ and Kuo-Chia Liu ${ }^{2}$ \\ NASA Goddard Space Flight Center, Greenbelt, MD, 20771
}

\begin{abstract}
The Solar Dynamics Observatory (SDO) spacecraft is a sun-pointing, semi-autonomous satellite that will allow nearly continuous observations of the Sun with a continuous science data downlink. The science requirements for this mission necessitate very strict sun-pointing requirements, as well as continuous ground station connectivity through high gain antennas (HGAs). For SDO's onboard attitude control system to successfully point the satellite at the Sun and the HGAs at the ground stations with the desired accuracy, in addition to the need for accurate sensors it must have good onboard knowledge of the ephemerides of the Sun, the spacecraft, and the ground station. This paper describes the minimum force models necessary for onboard ephemeris generation in support of an attitude control system. The forces that were considered include the Sun's point mass, Moon's point mass, solar radiation pressure (SRP), and the Earth's gravity with varying degree and order of terms of the geopotential.
\end{abstract}

\section{Introduction}

$\mathrm{T}$

HE Solar Dynamics Observatory (SDO) is the first mission to be launched for NASA's Living with a Star (LWS) Program, a program designed to understand the causes of solar variability and its impacts on Earth. SDO is designed to help us understand the Sun's influence on Earth and Near-Earth space by studying the solar atmosphere on small scales of space and time and in many wavelengths simultaneously.

The goal of SDO is to understand, driving towards a predictive capability, the solar variations that influence life on Earth and humanity's technological systems by determining:

- How the Sun's magnetic field is generated and structured

- How this stored magnetic energy is converted and released into the heliosphere and geospace in the form of solar wind, energetic particles, and variations in the solar irradiance

SDO is a sun-pointing semi-autonomous spacecraft that will allow nearly continuous observations of the Sun with a continuous science data downlink rate of 130 Megabits per second (Mbps). The spacecraft is 4.5 meters high and over 2 meters on each side, weighing a total of $3100 \mathrm{~kg}$ (fuel included). SDO will orbit at 36,000 km altitude in a circular geosynchronous orbit at 102 deg west longitude inclined at $28.5 \mathrm{deg}$. This orbit was chosen to allow continuous observations of the Sun and enable its exceptionally high data rate through the use of a dedicated ground station in White Sands, New Mexico.

In order to facilitate the needs of the science instrumentation, strict pointing requirements were placed on the attitude control system (ACS). These requirements necessitate onboard ephemeris generation as part of the ACS software. This paper details the design and validation of the ephemeris generation algorithm implemented in the ACS software.

\section{SDO Attitude Control}

The SDO attitude control system is a single-fault tolerant design. Its fully redundant attitude sensor complement includes 16 coarse Sun sensors, a digital Sun sensor, 3 two-axis inertial reference units, 2 star trackers, and 4 guide

\footnotetext{
${ }_{1}^{1}$ Aerospace Engineer, Flight Dynamics Analysis Branch, Code 595, AIAA Member

${ }^{2}$ Aerospace Engineer, GN\&C Systems Engineering, Code 591, AIAA Member
} 
telescopes. To maintain accurate Sun pointing, and to keep the spacecraft roll about the Sun vector correctly positioned with respect to the Solar North Pole, a three-axis stabilized ACS is used. ${ }^{1}$

The attitude determination function of the ACS flight software design is filled predominantly by an extended Kalman filter (EKF). To simplify attitude determination relative to the Sun itself, the software is designed to target quaternions in the body fixed frame and then convert them to an inertial reference frame based on onboard ephemeris calculations for the direction of the Sun relative to the spacecraft.

The SDO onboard ephemeris predicts the Earth Centered Inertial (ECI) locations of the Sun, Moon, spacecraft, and ground station. The ACS pointing error requirements were derived from the science requirements, the star tracker requirements, and the HGA pointing budget. The maximum pointing error from the spacecraft to the Sun, Moon, and ground station are $10 \mathrm{arcsec}, 1 \mathrm{deg}$, and $0.02 \mathrm{deg}$, respectively. These pointing requirements were used to calculate the necessary ephemeris knowledge requirements for the Sun, Moon, ground, and spacecraft, which are shown in Table 1.

\begin{tabular}{|l|c|c|c|c|}
\cline { 2 - 5 } \multicolumn{1}{c|}{} & SDO & Sun & Moon & Ground \\
\hline $\begin{array}{l}\text { Pointing Requirements } \\
\text { (from SDO) }\end{array}$ & N/A & 10 arcsec & $1 \mathrm{deg}$ & $0.02 \mathrm{deg}$ \\
\hline $\begin{array}{l}\text { Ephemeris Knowledge Requirements } \\
\text { (after 7 days) }\end{array}$ & $2.9 \mathrm{~km}$ & $3600 \mathrm{~km}$ & $3170 \mathrm{~km}$ & $2.9 \mathrm{~km}$ \\
\hline
\end{tabular}

Table 1. SDO Pointing and Ephemeris Knowledge Requirements. Pointing requirements were derived from science requirements, star tracker requirements and high gain antenna requirements. Ephemeris knowledge requirements were budgeted to each object by combining the pointing requirements.

Ephemeris knowledge requirements are derived based on the maximum position error allowable to meet the pointing requirements. For ephemeris error analysis, it is common to view the data in a coordinate system composed of radial, along-track, and cross-track components ${ }^{2}$. For our analysis, we looked at the radial error as well as the transverse error which we defined as the root-sum-square (RSS) combination of the along-track and cross-track components. Figure 1 diagrams how we define radial and transverse errors.

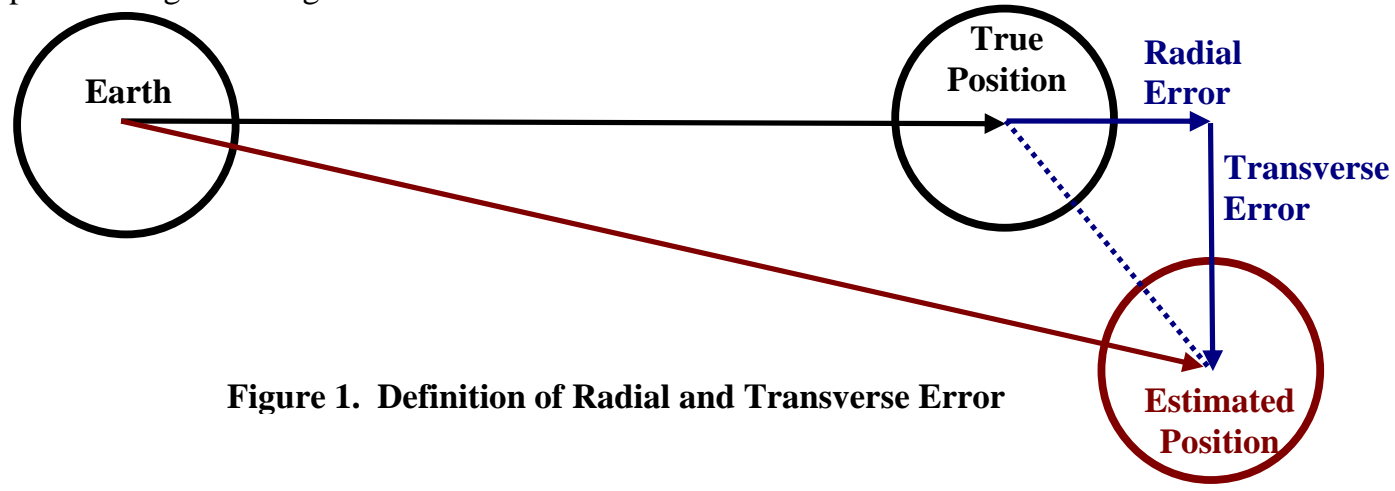

\section{Modeling Accuracies}

The SDO onboard ephemeris models were chosen based on the performance of previous missions such as the Tropical Rainfall Measuring Mission (TRMM) and the Hubble Space Telescope (HST). These models are used to predict the locations of the Sun, Moon, spacecraft, and ground station consistent with the requirements shown in Table 1. For added conservatism, ephemeris knowledge requirements were considered for a ten-day span instead of the required seven.

The ECI locations of the Sun and the Moon can be obtained very accurately by using the DE405 model developed by the Jet Propulsion Laboratory (JPL). However, this method would require either loading a large table of coefficients onboard the satellite and interpolating for each time step, or interpolating on the ground and uploading coefficients frequently. Neither of these options fills the need for a minimal model that can meet requirements for ten-days without updates.

The ECI locations of the Sun and Moon can be obtained very simply by the low precision formulae in the Astronomical Almanac ${ }^{3}$. This method meets requirements for the Moon, as will be shown later, but the precision of the Sun's apparent location is only good to $0.01 \mathrm{deg}$ or 36 arcsec. This formula does not meet the 10 arcsec pointing accuracy requirement defined in Table 1 .

American Institute of Aeronautics and Astronautics 092407 
Since the low precision formula is not accurate enough to meet requirements for the solar ephemeris, the calculation used is a numerical approximation obtained from Ref. 4. This approximation provides accuracy better than 2 arcsec during the entire ten-year SDO mission lifetime, which is far better than the 10 arcsec pointing requirements defined in Table 1 . Figure 2 shows the comparison between this model and the DE405 model. Radial and transverse errors were calculated over the ten-year mission lifetime to verify that the model always meets the $3600 \mathrm{~km}$ ephemeris knowledge requirement detailed in Table 1. Results are shown in Fig. 3.

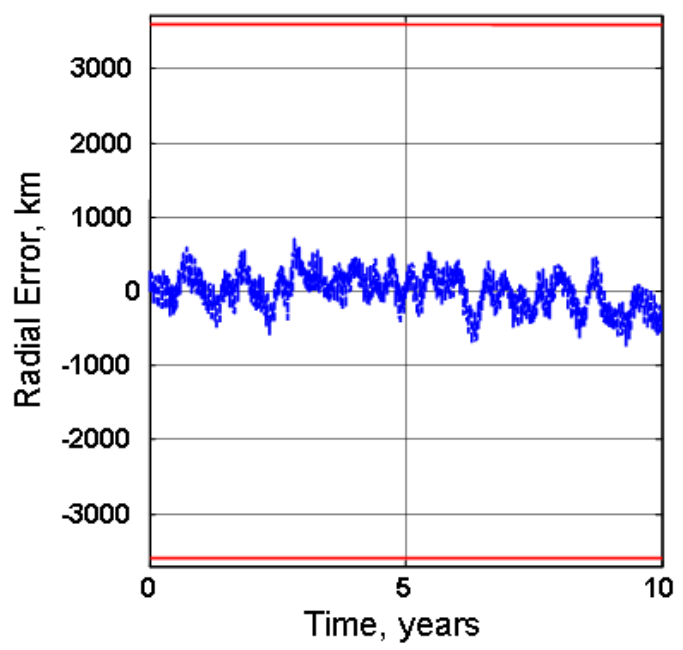

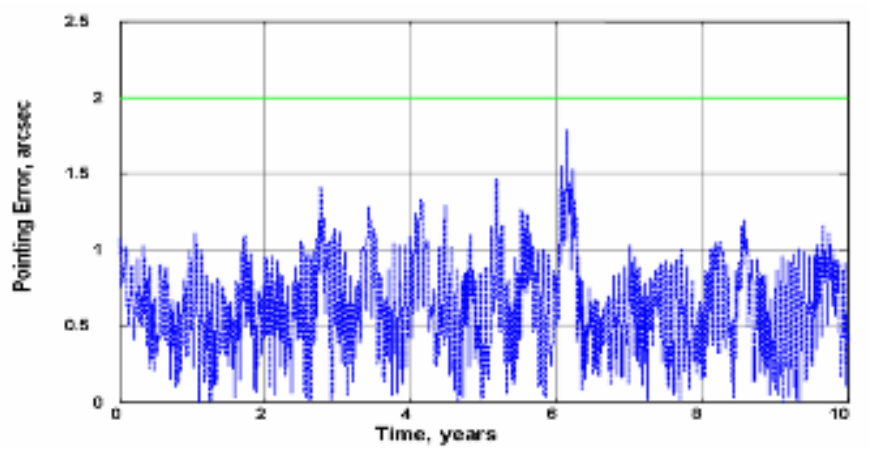

Figure 2. Solar Ephemeris Validation. Calculated Solar ephemeris validated against DE405.

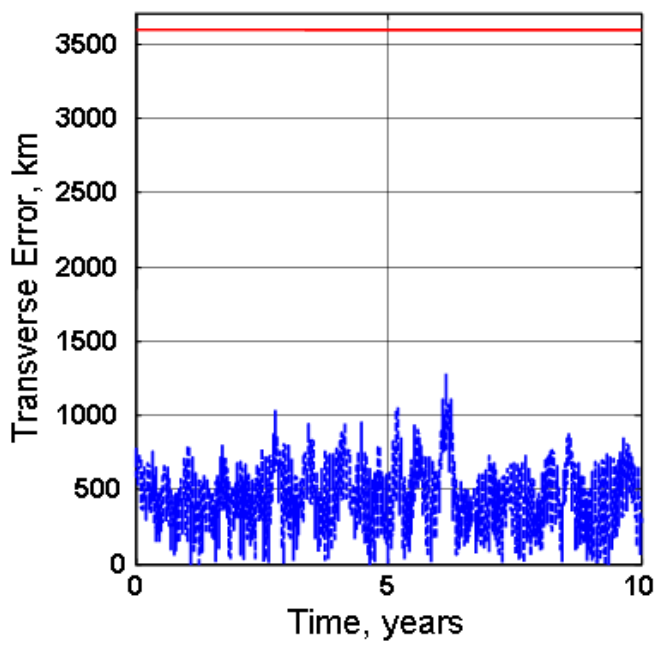

Figure 3. Radial and Transverse Errors in the Solar Ephemeris Calculation. Calculated Solar Ephemeris validated against DE405. Radial and transverse errors are calculated based on Fig. 1.

The lunar ephemeris calculation is based on the "Low-precision formulae for geocentric coordinates of the Moon" published in Ref. 3. This algorithm was chosen because of its simplicity and its successful implementation on HST. Figure 4 shows the comparison between this model and the DE405 model. The models agree to within 0.36 deg over the entire ten-year mission lifetime, which is well within the 1 deg pointing requirement in Table 1 . Radial and transverse errors were calculated over the tenyear mission lifetime to verify that the model always meets the $3170 \mathrm{~km}$ ephemeris knowledge requirement detailed in Table 1 . Results are shown in Fig. 5.

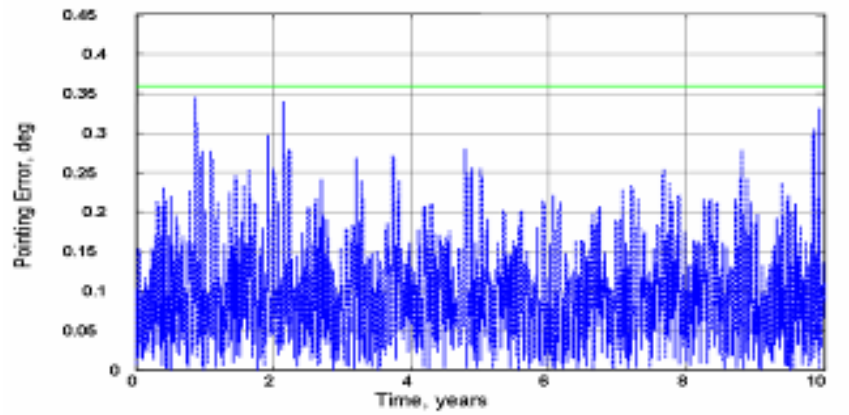

Figure 4. Lunar Ephemeris Validation. Calculated Lunar ephemeris validated against DE405. 

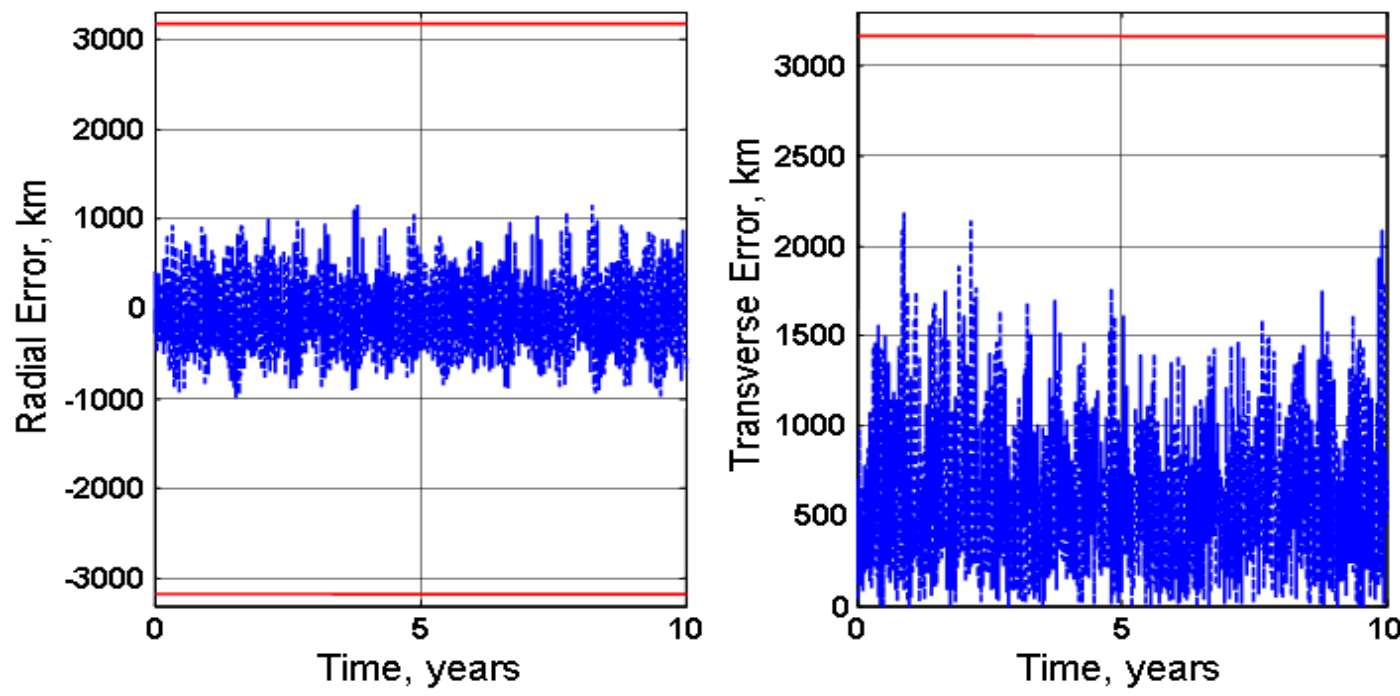

Figure 5. Radial and Transverse Errors in the Lunar Ephemeris Calculation. Calculated Lunar Ephemeris validated against DE405. Radial and transverse errors are calculated based on Fig. 1.

The spacecraft ephemeris is generated by numerical integration of a force model using a standard fourth-order Runge-Kutta routine. The force model that was chosen for SDO's onboard ephemeris generation includes several geopotential terms, Sun and Moon point mass gravitational effects, and the solar radiation pressure effects. The validation of this force model is included in the next section.

The acceleration due to gravity is defined by the gradient of the potential of the Earth (the geopotential). A common model of the Earth's gravity field is a spherical harmonics model based on Legendre polynomials. One such model is the Joint Gravity Model; the second version is called JGM2, which describes perturbations to the Earth's gravity field up to degree and order 70. With this type of series expansion, however, the contribution of each term tends to become smaller as the degree and order are increased, and not all terms are necessary for all satellite missions. The perturbations on the gravity field are divided into zonal, sectorial, and tesseral harmonics ${ }^{2}$.

The zonal terms of the geopotential model divide the perturbations due to the non-uniform mass distribution into varying bands of latitude. The $\mathrm{J} 2$ term divides the globe into 3 bands and accounts for the equatorial bulge; the J3 term divides the globe into 4 bands and accounts for the globe's pear shape; and so on. Figure 6 shows the magnitude of the maximum acceleration contributed by each of the first five zonal terms on a logarithmic scale for circular orbits ranging from low Earth to geosynchronous. A constant acceleration changes the position of a body by $1 / 2 \mathrm{a}^{*} \mathrm{t}^{2}$, so a constant acceleration of $7.8 * 10^{-9} \mathrm{~m} / \mathrm{s}^{2}$ would produce a position change of $2.9 \mathrm{~km}$ after ten days. This simple calculation suggests that any terms adding acceleration less than $10^{-10}$ would not need to be modeled to meet our requirement. Thus, J2, J3, and J4 were included in SDO's geopotential model.

The sectorial terms of the geopotential model divide the perturbations due to the non-uniform mass distribution into varying wedges of longitude. The C22 and S22 terms divide the globe into two hemispheres, and their contribution produces a resonance effect. Based on previous geosynchronous missions, the C22 and S22 terms need to be modeled, but any subsequent terms can be ignored. For completeness, this statement will be validated in section IV.

The tesseral terms of the geopotential model divide the perturbations due to the non-uniform mass distribution into varying dimensions of tiles across the globe. Based on previous geosynchronous missions, these terms do not need to be modeled. For completeness, this statement will also be validated in section IV.

The resulting Earth gravity model chosen for SDO includes the acceleration caused by the Earth as a point mass, as well as the perturbations due to J2, J3, J4, C22, and S22. A complete validation of this model was performed comparing results to the Satellite Tool Kit (STK®) developed by Analytical Graphics Incorporated and the Goddard Trajectory Determination System (GTDS). This validation is described in part B of section IV. 


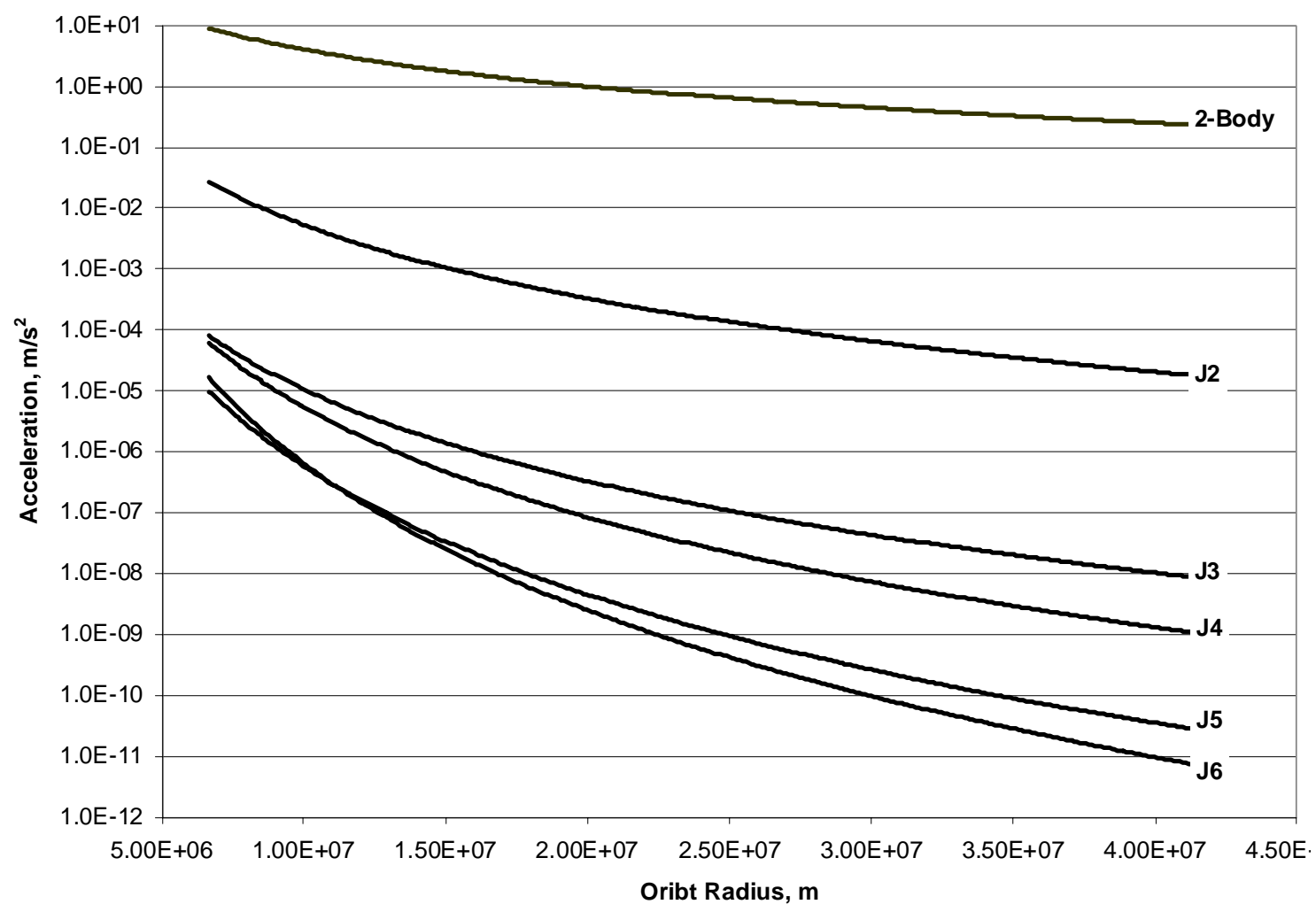

Figure 6. Maximum Acceleration Added by Each Zonal Term. Each term was calculated individually at the latitude that provided the largest acceleration for each orbit radius from low earth orbit to geosynchronous. Since the accelerations are plotted on a log scale, this figure provides an order of magnitude comparison for each of the first five zonal terms of the geopotential model.

The ground station location is calculated by a transformation from Earth Centered Earth Fixed (ECEF) coordinates to Earth Centered Inertial (ECI). The station location is obtained from the World Geodetics System (WGS-84) version of the NASA Directory of Station Locations (NDOSL) in ECF coordinates. Since the satellite ephemeris is generated in ECI coordinates, the location must be transformed by a time dependent matrix rotation. The full ECF to ECI transformation is calculated based on the Greenwich Hour Angle (GHA), precession, nutation, and polar motion. Since nutation and polar motion account for less than 9 arcsec and 0.6 arcsec of change respectively ${ }^{5}$, they can be ignored for our purposes. The resulting rotation matrix composed of GHA and precession calculations was used to rotate the NDOSL station location and is validated against a complete model using the FreeFlyer ${ }^{\circledR}$ software developed by a.i. Solutions. Radial and transverse errors were calculated over the ten-year mission lifetime to verify that the model always meets the $2.9 \mathrm{~km}$ ephemeris knowledge requirement detailed in Table 1. Results are shown in Fig. 7.
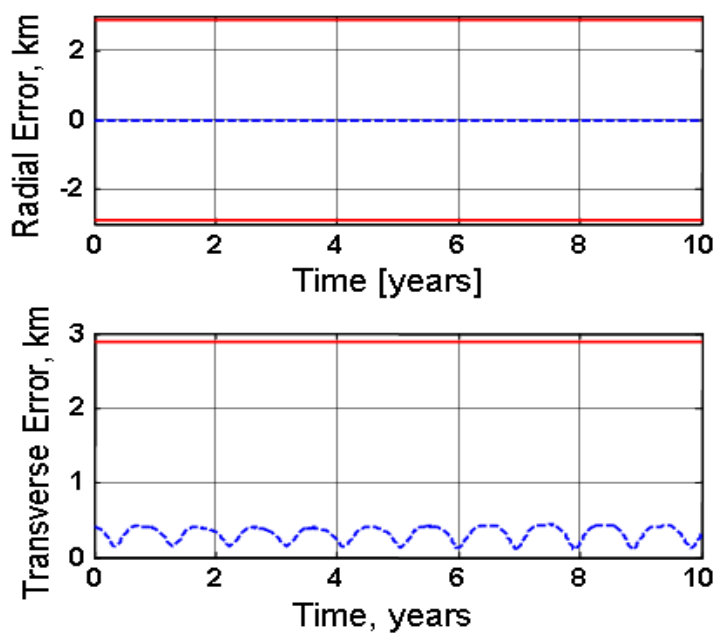

Figure 7. Ground Station Ephemeris Validation. ECI Rotated ground station ephemeris validated against FreeFlyer. Radial and transverse errors are calculated based on Fig. 1. 


\section{Force Model Validation}

\section{A. STK Force Model Comparisons}

The previous section described the reasoning for choosing each term in the SDO force model. This section will show the errors associated with several different combinations of those terms. At SDO's geosynchronous altitude, propagating a 12x12 geopotential for ten days produces an ephemeris that agrees with the $70 \times 70$ to within 20 micrometers, which implies that a full 70x70 geopotential model is unnecessary for our analysis. For validation purposes, the "truth" model used included a 12x12 geopotential, Sun, Moon, and Solar Radiation Pressure (SRP). The integrator used in STK is an $8^{\text {th }}$ order Runge-Kutta.

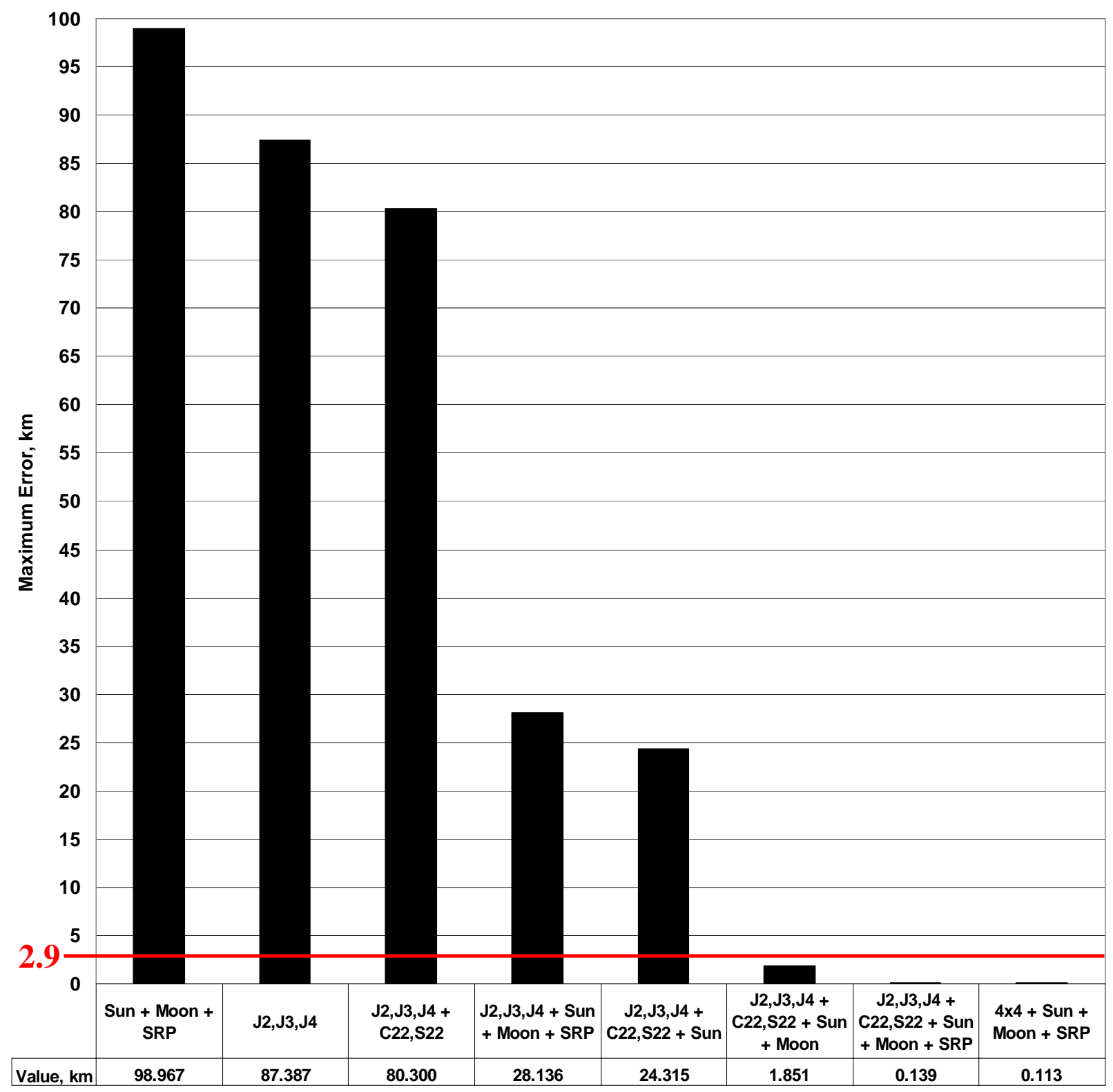

Figure 8. Position Errors Caused by Different Modeled Forces. Each bar represents the maximum position error after a ten-day propagation of the SDO orbit with Earth's point mass gravity plus the acceleration due to the terms listed. Several different combinations of Sun, Moon, SRP, the $1^{\text {st }}$ three zonal geopotential terms, and the $1^{\text {st }}$ sectorial geopotential term where examined to find the minimum set of terms needed. This graph suggests that requirements are met without SRP calculations, but the integrator used onboard introduces more error that is not shown here. This graph also shows that using a $4 \times 4$ geopotential model does not increase performance by much.

American Institute of Aeronautics and Astronautics 092407 
Since SDO will be in orbit around the Earth, there is no question that Earth's gravity will affect the satellite's position. In addition to Earth's point mass gravity, we wanted to analyze the effects of adding the Sun's point mass, the Moon's point mass, SRP, the 1st three zonal geopotential terms (J2,J3,J4), and the 1st sectorial geopotential term (C22,S22). Figure 8 shows the errors in SDO's orbit after propagating for ten days with different combinations the terms just listed.

It should be noted that this analysis suggests that the full SDO force model may not be the minimum set required to meet the requirements. The $3^{\text {rd }}$ to last bar in Fig. 8 shows that SRP is not required for STK to produce a result that has less than $2.9 \mathrm{~km}$ of error after ten days. However, when this SRP-free model is used with the $4^{\text {th }}$ order RungeKutta integrator that is implemented onboard SDO the error grows near the $2.9 \mathrm{~km}$ limit. Since SRP is a simple calculation, the addition of the extra line of code is well worth the large increase in performance and allows us to anticipate a possible need for better ephemeris knowledge.

As a final test, a $4 \times 4$ geopotential model was analyzed and the results didn't provide a significant increase in performance. This validates our previous assumption that all sectorial term other than C22 and S22, as well as all tesseral terms, can be ignored in our force model.

\section{B. Validation Against STK and GTDS}

The full SDO force model was compared to the "truth" model in both STK and GTDS. The SDO force model was propagated in the SDO flight software written in C with a $4^{\text {th }}$ order Runge-Kutta. The STK "truth" was propagated with an $8^{\text {th }}$ order Runge-Kutta and the GTDS “truth" was propagated with a $12^{\text {th }}$ order Cowell integrator. Errors in this validation include differences in integration algorithms, errors in the force model, and errors due to the Sun and Moon ephemeris approximations described in this paper. Figure 9 shows the radial and transverse errors in SDO's position against GTDS (in red) and STK (in blue). Both validations show that the SDO flight software meets the ephemeris knowledge requirement of $2.9 \mathrm{~km}$.
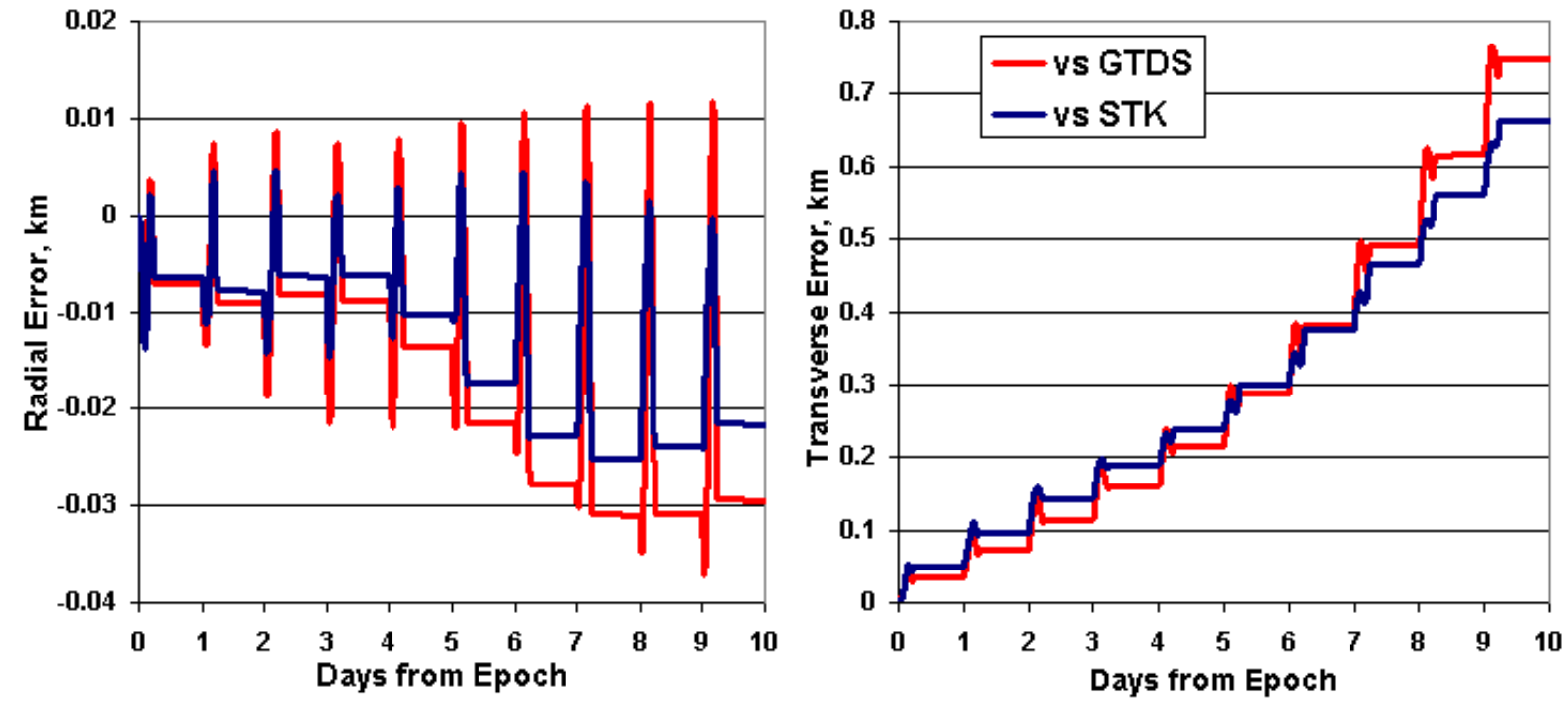

Figure 9. Radial and Transverse Errors in the Satellite Ephemeris Calculation. The SDO flight software is compared to GTDS and STK, which are both validated software packages. Radial and transverse errors are calculated based on Fig. 1.

\section{Conclusion}

The selection of models for onboard ephemeris generation involves a trade-off between simplicity and accuracy. Simple models put less of a strain on the onboard computer, but model accuracy must be good enough to meet requirements. The models presented in this paper were chosen for their simplicity, while ensuring that sufficient accuracy is provided to meet the requirements for the SDO onboard ACS filter. The validation for each model compared the ephemeris generation of the Sun, Moon, spacecraft, and ground station to very accurate models ("truth" models) and showed that all requirements are successfully met. 


\section{References}

${ }^{1}$ Starin, Scott, et al., “Attitude Control System Design for the Solar Dynamics Observatory”, NASA, Goddard Space Flight Center SDO MIS Document 464-ACSREF-0129, October 2005

${ }^{2}$ Vallado, David A., Fundamentals of Astrodynamics and Applications, $2^{\text {nd }}$ ed., Space Technology Library, El Segundo, California, 2001, pp. $509-552$.

3“The Astronomical Almanac”, U.S. Government Printing Office, 1994.

${ }^{4}$ Bretagnon, Pierre, "Planetary programs and tables from -4000 to +2800 : Tables for the motion of the sun and the five bright planets from -4000 to +2800”, Willmann-Bell, 1986.

${ }^{5}$ Tapley, Byron D., Schutz, Bob E., Born, George H., Statistical Orbit Determination, Elsevier Academic Press, Burlington, MA, 2004, pp $71-82$. 